\author{
AUthor(S): C. Y. Huang, S. A. Wolf, C.! W. W. Fuller, \\ J. $H$ ang, and C. S. Ting
}

\author{
SUBMITTEO TO: Invited paper \\ Proceedings of the 9th AIRAPT International High Pressure Conference \\ 24-29 July 1983, Albany, NY
}

\title{
IDIS' 'I.AIMF.R
}

This reporl was prepured us un a cou 11 ol work spmonsored hy un ugency of the I/nited States (iovernment. Neilher the U/nited Siutes (wovermument nu, uny ngency thereof, nor any of their employees, mukes uny warranty, express or implied, w1 ussumes any lequl liuhility or renponsibility for the Heciuracy. commletenesss, or usefulness of any informution, uppuratun. product, of proceen disclosed, or represents thill its use would not infringe privulely owned rights. Refer-

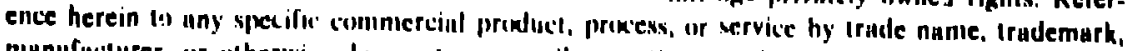
munufucturer, or wherwise deses not necessirily constitute or imply its endorsement, recommendation, or linvoring by the I/niteal Stittes (iuvermuent or any ukency thereat. The view,

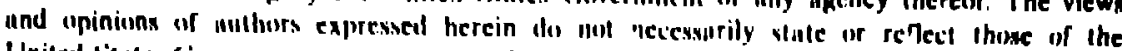
United Situles rinvernment or any aneney therenol. 
INTERPLAY BETWEEN SUPERCONDUCIIVITY AND ITINERANT FERROMAGNETISM IN $\mathrm{Y}_{9} \mathrm{CO}_{7}$ AT HIGH PRESSURE

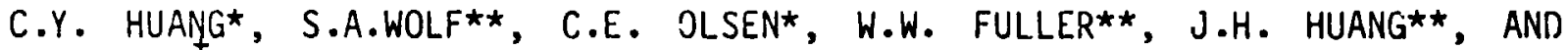
C.S. TING

*Center for Nonlinear Studies and Physics Div., Los Alamos National Laboratory, Los Alamos, NM 87545; **Code 6634, Naval Research Laboratory, Wash. DC 20375; Physics Dept., Univ. of Houston, Houston, TX 77004

The theory of the interplay between superconductivity and itinerant ferromagnetisin based on the single conduction band model in the mean field approximation is summarized. This theory predicts that superconductivity and itinerant ferromagnetism cannot co-exist, that the nigher temperature magnetic phase can transform into the low temperature superconducting state, and that a superconducting state will not transform into a ferromagnetic state at a temperature below the superconducting transition. For the magnetic superconductor $\mathrm{Y}_{9} \mathrm{CO}_{7}$ we have reviewed the experimental results and in particular, we have shown that pressure suppresses magnetism rasulting in a higher superconducing transition temperature and have concluded that $\mathrm{Y}_{9} \mathrm{CO}_{7}$ is an itinerant magnet. Pressure also sharpens the superconducting transition and increases the upper critical field, signifying that the ferromagnetic correlations and superconducting fluctuations co-exist but very spatially. For pressures greater thar, 5 kbar, the magnetoresistance is always positive, further indicating the suppression of magnetism by high pressure. Several microscopic experiments and some improvement in theory are sugyested.

\section{INTROOUCTION}

For the past several years the interplay between super inductivity and indgnetism has attracted considerable interest.[1-3] To date most of the studies, experimental as well as theoretical, have concentrated on the rare cartin ternaries whose particular crystal structure gives rise to weak magnetic interactions between the localized ind gnetic moments arising from $4 f$ rare earth ions and the superconducting d-electrons of the transition metal. i.e. separate electrons are responsible for superconduct ivity and inagnet ism, respective!y.[3] Recen:ly a new intermetallic magnetic superconductor, $\mathrm{Y}_{9} \mathrm{CO}$, has been intensively investigaied.[4-14] In this compound, in contrast to the well-established ternary superconductors, majnetism is itin.. erant in nature and superconductivity appears at $T, 1,3 K$ ) which is be low the temperature at which the compound becones magnetic $\left(\mathrm{T}_{\mathrm{m}}, \mathrm{.6 \textrm {K }}\right)$. The measurement.s on this compound have yielded interesting result's replete with anomalies. The detailed experimental investlgations have concluded that ferromagnitic correlations whtch develup at higher temperatures remain finite and that malgnetisill is confined by superconductivity. Neutron depolarization studles have led to the conclusion of the presence of a "hybrid" state with the inhomogeneous spacial variation of the order paramecer.

In our previous studies, we have show: that unusud lermi surfaces are very sensitive to external pressure, [1b] and that itinerant ina gnetisill could be easily suppressed by high pressure. [10] furthermore, owiny to the fact that the application of high pressure is a useful approach to study 
the interplay between superconductivity and magnetism,[17] the electrical resistance and the magnetic susceptibility in $\mathrm{Y}_{9} \mathrm{Co}_{7}$ have been measured up to 20 kbar. [14] The experimental results have led to the conclusion that high pressure suppresses the magnetic correlation raising the superconducting transition temperature $T_{s}$.

In this paper, we briefly describe the theoretical asfect of the problem based upon a single-band conduction election model in a mean field approximation. We also discuss some salient features of the current experimental results. Finally, the experimental results at high pressure (up to $20 \mathrm{kbar}$ ) and at high magnetic field (up to $6 \mathrm{~T}$ ) will be presented.

\section{SUMMARY UF THEORETICAL RESULTS}

The theoretical aspects of the interplay between itinerart ferromagnetism and supercondustivity were first investigated by Nakanishi, et al [18] employing the mean field approximation. They studied the interchange of the itinerant ferromagnetic and superconducting phases. Very recently, Lei, et al[19] have calculated the free energies at the same level of approximation by using a single-band model in which ferromagnetism and superconductivity arise from the same electrons. The superconducting part was treated by the BCS theory and the ferromagnetic part made use of the Hartree-Fock approximation. These authors have shown that the co-existent state in which itinerant ferromagnetism and superconductivty co-exist uniforinly in space has the highest free energy and is thus unstable.

Therefore in this model, the co-existent state cannot appeir as a thermodynamically stable state. They have also found that the stability of the ferromagnetic state against the superconducting state depends on the parameters involved. When the parameters are chosen so that the ferromagnetic transition temperature in the absence of superconductivity, $T_{m o}$ is nuch higher than the superconducting transition temperature in the mobsence of maynetisin, $T_{s o}$, the free energy of the ferromagnetic state is lower than that of the superconducting state for $T<T$, and the state of the system is ferromagnetic; superconductivity does not appear. For $T$ higher, but

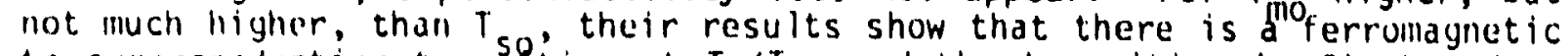
to superconducting transition at $T_{s}<T$ and the transition is first order. Figure 1 displays the temperature dependence of the ferromagnetic order (magnetization). $M$, and the superconducting order, $\Delta$, under this situation.

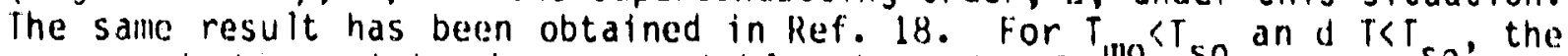
superconducting state is more stable than the ferllomaghetic state, and therefure ferromagnetisin cannot re-enter once the superconductivity is a lready present. Thiese results are quite contrary to the re-ent.rant ferromagnetic ternartes [3] where superconductivity occurs at higher temperature followed by the appearance of ferromanet ism at lower temperature. In these teernaries terromagnetisin arises from the localized $4 \mathrm{f}$-electrons while superconductivicy comes from the d-electrons and the free energies of these two ordered states can be regarded independent of each other and they can take whatever vo !ues the systeill allows them to have.

It should be noted that the single-band model may not be applicable to a real system. There has been speculation chat in $Y_{\text {glo }}$, the halelectrons of $Y$ are responsible for superconductivity and the 3ep-e liectrons of (i) for Indynetism. In this case a inere sophisticated theory than the single-band model I is needed. Moreover. the mean field approximation deses not take into consideration superconduct iny fluctuations. Thereture, the co-existance of superconducting fluctuations with itherant ferromalyetism above $r$, as suggested by sume experlmental results 10.111 doe" not contradic the present theory. 


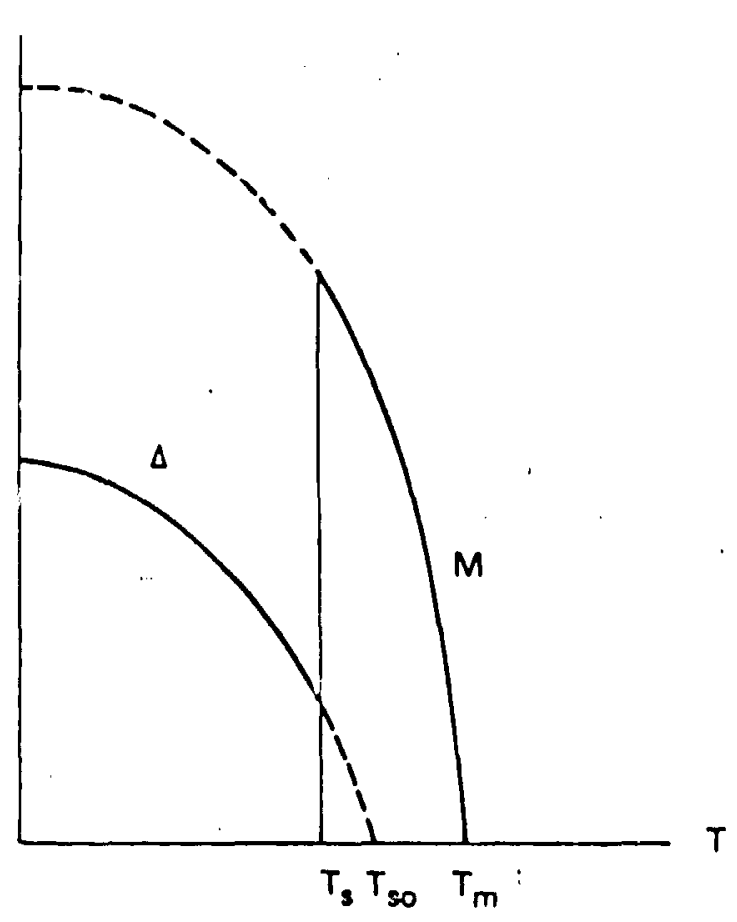

Fig. 1 Temperiture dependencies of the superconducting order-parameter, $\Delta$ and the magnetization, $M$, when the magnetic transition temperature, $T_{m}$, is higher than the superconducting transition temperature, $T_{S}$. Here $T_{s}$ is 'ower than $T_{S 0}$ the trahsitions iemperature when magnetisin is absent.

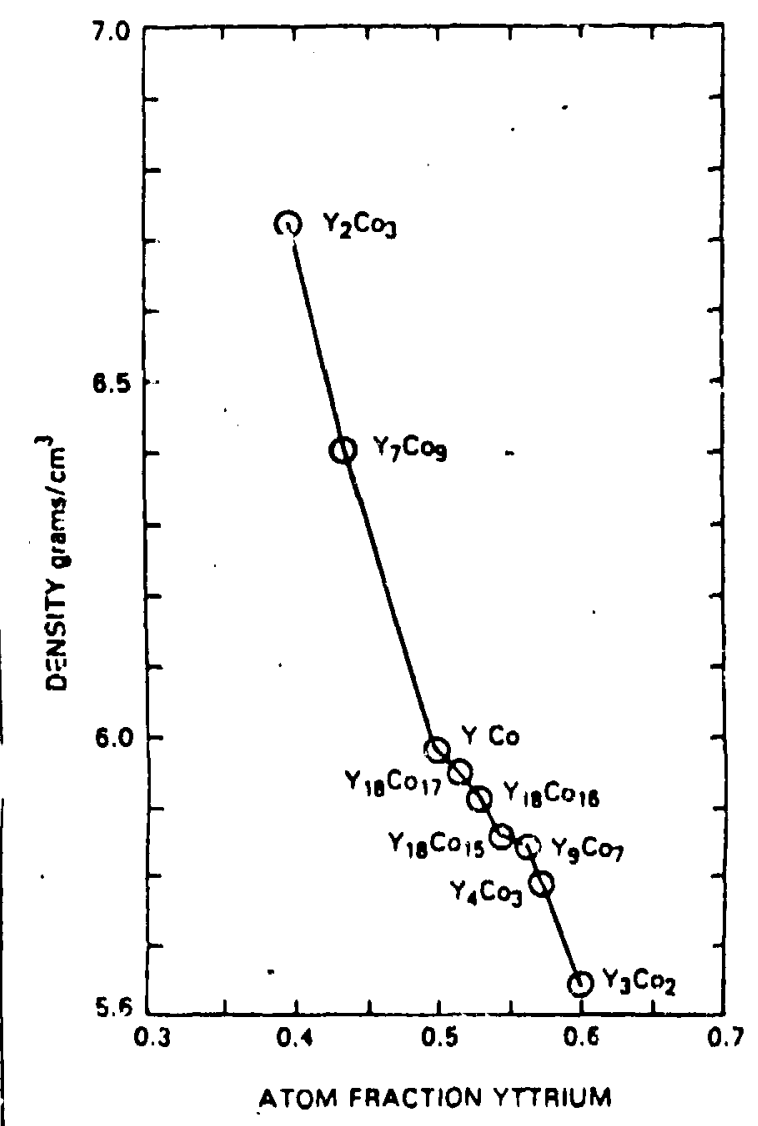

Fig. 2 Diagram of density of alloys vs yttrium itom fraction.

SAMPLES

$\mathrm{Y}_{9} \mathrm{CO}_{2}$ crystalli es with an hexagonal unit cell [20] resulting from the assembly of $Y$ atc is in the $f(\mathrm{~mm}$ of trigonal prisms. A group of rour $C O$ atoms is enclosed within the center of each of the $Y$ prisms. The remainder of the co atoms lie a! ong the c.axis of the unit celi. From the detailed study of this compound $[10,11]$ sone properties seem to be sensitive to the inhomogeneity and purity of the sdipple. The nccurrence of suparconductivity and magnetisil depends strongly on the i, $\mathrm{CO}_{7}$ crystal structure. The phase diagram of the $Y$-Co binaries has been investigated by Grover, et al [21]. Their results show the existence of a single phase over a narrow composition range of $\mathrm{Y}_{9} \mathrm{CO}$. . For the $\mathrm{Y}_{9} \mathrm{CO}_{3}$ composition which was originally studied and led to the discovery of $Y_{9} \mathrm{Cu}_{\%}$, the hexayonal $Y_{9} \mathrm{Co}$, phase persists with $Y_{8} C_{C O}$ as an impurity phase in which $Y_{8} \mathrm{CO}_{5}$ is rot superconducting and shows nó maynetic ordering.

Whon our work was initialed, the only published $Y$-C.o phase diagram was that of Ray.[22] farly work on the magnet ic and superconducting propertiles in this system indicated that there was a question as to the compound consposition of thr superconduct iny phase. Becadse compound occurrencescan be determined as a function of composition by the breaks in density vs composition diayrams. a serios of alloys was prepared and heat treated for 336 hours at $5300^{\circ}$ C. The alloy dens it ies were determined by weighing the butcons in bromobonache. The rosults of these detcerminations are plotied in fig. 2.

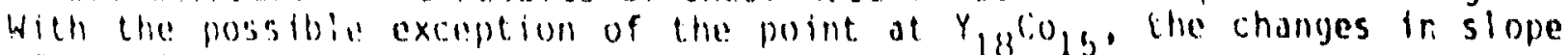
of density vs yttrium atom fraction at $Y_{2} \mathrm{CO}_{2}$, PCO, and $Y_{0} \mathrm{CO}_{\mathrm{O}}$ are consistent with the now phase didyram published hy Grover et al.[21] As a result, 
$\mathrm{Y}_{9} \mathrm{CO}_{7}$ appears to exirt as a single phase.

PRL 'IS EXPERIMENTAL RESULTS

jure 3 shows the results of the ac magnetic susceptibility, $x$, arid electr. al resistance, $R$, obtained by Sarkissian.[11] In this sample superconductivity sets in at $\approx 3 K$ and the magnetic transition takes place at, $6 K$. It is ev aent that themal .ysteresis is present in the magnetic region. This author has also shown the current dependence of the electrical resistance in the magnetic region, [10] strongly indicating that superconducting interactions co-exist with strong magnetic correlation. In addition, he has measured the magnetoresistance in the magretic and superconducting state. At low temperature but above the superconducting transition, the low field magnetoresistance indicates that superconducting fluctuations are present. This obseivation further suggests that the superconducting interactions are present in the magnetic region.

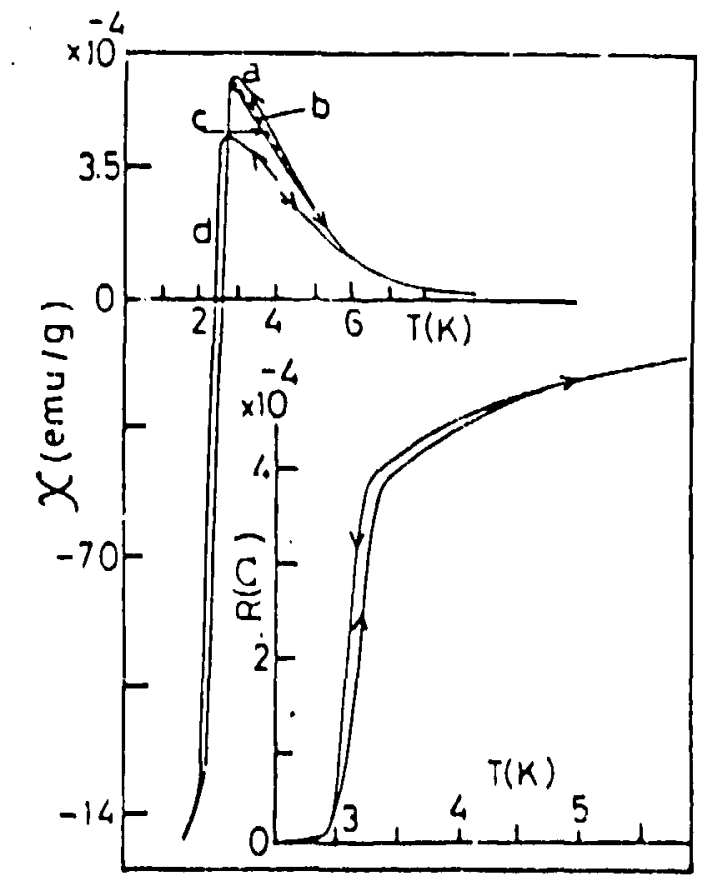

Fig. 3 Temperature dependence of the ac suscrotibility, $X$, in $\mathrm{Y}_{9} \mathrm{CO}_{7}$. The arrows indicate in which direction the temperature was varted. a) cooling down from $4.2 \mathrm{~K}$ to $1.5 \mathrm{~K}$, b) and c) second and third warming cycles and d) $x$ in the same sample before further low temperature annealing. The inset shows the resistance data. (From Ref. 11).

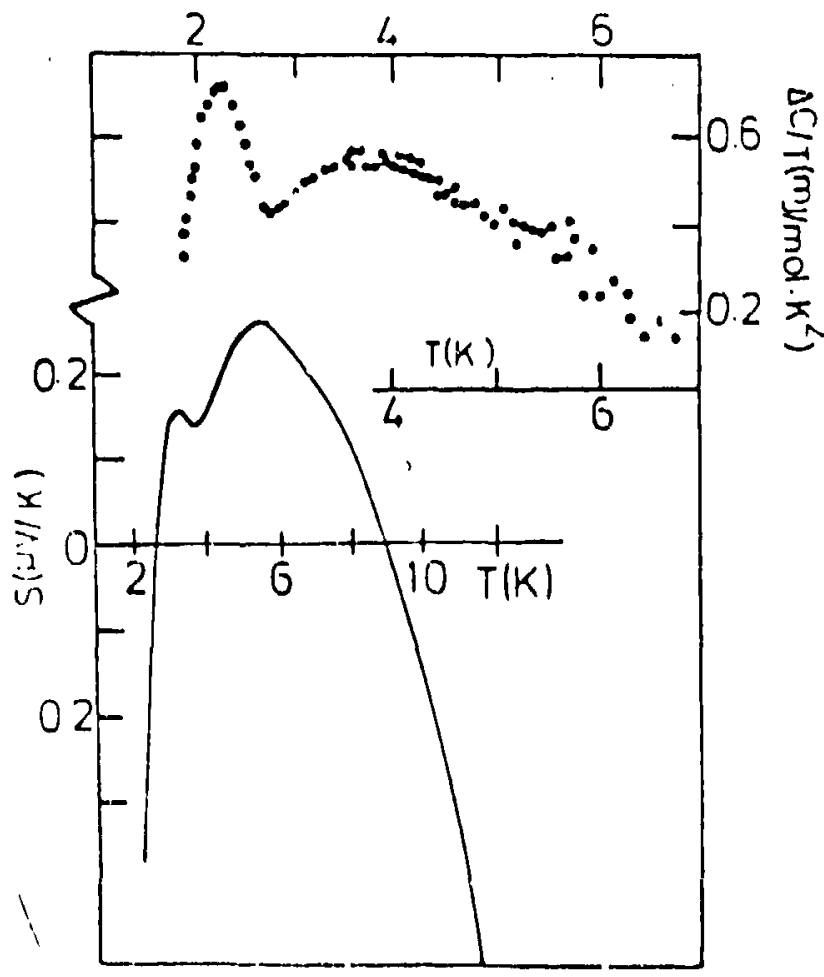

Fly. 4 Excess heat capactity $\Delta C / T$ and thermopower $\mathrm{S}$ of $\mathrm{Y}_{9} \mathrm{CO}_{7}$. (From Ref. 11).

In addition to the transport properties. Sarkissian has measured the magnetization in maynetic fields from Hi, L Gaus to 200 Gauss. [10,23] According to his data, there is no sign of lirear behavior in $M^{2}$ and $M / l_{1}$ plots in the low field reyime. The maynet ic susceptibility at high trmperatures does noi follow a Curie-Weiss law but rather tonds to temperatureindependence, as expected for an itinerant magnet. This result is consistent with the obicrvation that the cemperature dependence of the high temperature resistance is less than a linear rise with temberature. Moreover, his M-ll hysteres is loops, taken in fielits up to 200 liauss, have enabled hill to observe the Meissner effect. From his observation that the hysteres is loop 
is more pronounced be low $T_{S}$, he has concluded that the hysteres is is, at least partly, due to superconductivity not just ferromagnetic domain walls as in an ordinary magnet.

The heat capacity and thermopower are the best demonstration of bulk superconductivity. Figure 4 from Ref. 11 shows the excess heat capacity $\Delta C / T$, after substracting lattice and electronic contribution. The peak at $22.5 \mathrm{~K}$ clearly displays the bulk superconducting transition. The peak in the thermopower, $S$, around $3 K$ is a lso consistent with this transition.

Among the salient features in $Y_{9} \mathrm{Co}_{7}$, the results of the depolarization of polarized neutrons $[10,11]$ are partitularly interesting. Sarkissian has shown that the depolarization is present only when the magnetic field is applied perpendicular to the incident neutron polarization. The abrupt onset of depolarization takes place below 4K, displaying the kind of behavior to be expected if inhomogeneities were forming in the temperature region in which magnetic correlations are present. This inhomogeneity is consisient with the "hybrid" state present in the magnetic region discussed above. The finite depolarization below $T$ has been observed, but, because of the possible depolarization originating from the flux line domains, the question of complete or partial suppression of viagnetic correlations could not be answered.

\section{EXPERIMENTAL. RESULTS}

The $\mathrm{Y}_{9} \mathrm{CO}_{7}$ samples used in our high pressure experiments were prepared by Johnson, Matthy and Co. $99.999 \%$ pure cobalt and $99.99 \%$ yt.trium from the Research Chemical Co. The alloys were made by arc malting together weighed amounts of cobait and yttrium metals on a water-conled copper hearth in a gettered argon atmosphere. The buttons were turned and remelted ten times to insure compositional homogeileity. Melting 1.3ss as did not exceed $0.4 \%$ so that the compositions were taken to be those as veighed out. The samples were heat treated for 20 days at $530 \pm 5^{\circ} \mathrm{C}$ in a high vacuum furnace. Chemical analysis, within limits of the atomic adsorption method used, shows the material to have the composition $Y_{9} \mathrm{Co}_{7}$. Powder $x$-ray diffraction patterns were taken $n$ l the samples. All the observid lines could be indexed on the structure, "Y $\mathrm{CO}_{3}$ " reported by Lamaire, et $21 .[20]$ Within the sens $\mathrm{i}-$ tivily ( $25 \%$ ) the alloys at $\mathrm{Y}_{9} \mathrm{CO}_{7}$ were single phase. The resistivity ratio $\left(R_{3 \cup 0} / K_{4.2}\right)$ of oui samples is 216 , ccmparable with others. $[10,11]$ The $T$ of our sarples is ahout $0.5 k$ lower than those used in Refs. 10 and 11 possibly because of the presence of Gd impurities ( 100 ppill) in yttrium. However, this lower $T_{c}$ should not se iously effect our high pressure sludies.

We have simultaneously measured the electrical resistance and ac magnetic susceptibility of the samples as a function of temperature from $11.2 \mathrm{~K}$ :0 300K, at pressures up to $1,20 \mathrm{kbar}$ and magnetic fields up to 6T. The resistance was measurerd uring a standard four-terminal ac technique at $33 \mathrm{~Hz}$, while a $610 \mathrm{~Hz}$ susceptibility signil was ubtained from a secondary coil wrapped directly arcund the sample. The pressure was generated in a 1:1 flute mixture of n-pentane and isoamyl alchol contained inside a 3.2. Imm thameter Teflon cup enclosed inside a berylium-copper cell, enploying the self-clamped technique. The pressure was monitored by a lead maremeter with an acciracy of $t .1$ kbar. A superconducting magnet capable of yeneracing a magnetic field up to 61 was employed for the machet ic field studies.

The temperature dependence of the ac magnetic susceptiblility at various

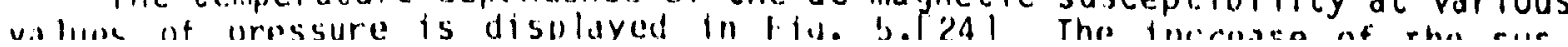


is more pronounced bel ow $T_{S}$, he has concluded that the hysteres is is, at least partiy, due co superconductivity not just ferromagnetic domain walls as in an ordinary magnet.

The heat capacity and thermopower are the best demonstration of bulk superconductivity. Figure 4 from Ref. 11 show, the excess heat capacity $\Delta C / T$, after substracting lattice and electronic contribution. The peak at $\sim 2.5 \mathrm{~K}$ clearly displays the bulk superconducting transition. The peak in the thermopower, $S$, around $3 K$ is also consistent with this transition.

Among the salient features in $Y_{9} \mathrm{Co}_{7}$, the results of the depolarization of polarized neutrons $[10,11]$ are particularly interesting. Sarkissiar has shown that the depolarization is present only when the magnetic field is applied perpendicular. to the incident neutron polarization. The abrupt onset of depolarization takes $p l a c e$ below 4K, displaying the kind of behavior to be expected if inhomogeneities were forming in the temperature region in which magnetic correlations are present." This inhomogeneity is consisient with the "hybrid" state present in the magnetic region discussed above. The finite depolarization below $T$ has been observed, but, because of the possible depolarization originating from the flux line domains, the question of complete or partial suppression of magnetic correlations could not be answered.

\section{EXPERIMENTAL RESULTS}

The $\mathrm{Y}_{9} \mathrm{CO}_{7}$ samples used in our high pressure experiments were prepared by Johnsun, Matthy and Co. $99.999 \%$ pure cobalt and $99.99 \%$ yttrium from the Research Chemical Co. The alloys were made by arc melting together weighed amounts of cobalt and yttrium metals on a water-cooled copper hearth in a gettered argon atmosphere. The buttons were turned and remelted ten times to insure compositional homogeneity. Melting losses did not exceed $0.4 \%$ so that the compositions were taken to be those as weighed out. The samples were heat treated for 20 days at $530 \pm 5^{\circ} \mathrm{C}$ in a high vacuum furnace. Chemical analysis, within 1 imits of the atomic adsorption method used, shows the material to have the composition $\mathrm{Y}_{9} \mathrm{CO}_{7}$. Powder $x$-ray diffraction patterns were taken on the sampies. All the observed lines could be indexed on the structure, "Y $\mathrm{CO}_{3}$ " reported by Lamaire, et al.[20] Within the sensitivity $(\sim 5 \%)$ the ailoys at $\mathrm{Y}_{9} \mathrm{CO}_{7}$ were single phase. The resistivity ratio $\left(R_{300} / R_{4,2}\right)$ of our samples are $\sim 16$, comparable with others.[10,11] The $T$. of our samples are about $0.5 \%$. lower than those used in Refs. 10 and 11 possibly becalse of the presence of Gd impurities ( $100 \mathrm{ppm}$ ) in yttrium. However, this lower $T_{c}$ should not seriously effect our high pressure studies.

We have simultaneously mearured the electrical resistance and ac magnetic susceptibility of the samples as a function of temperature from $\sim 1.2 \mathrm{~K}$ to 300K, at pressures up to $20 \mathrm{kbar}$ and magnetic fields up to $6 \mathrm{r}$. The resistance was measured using a standard four-tenminal ac technique at $33 \mathrm{~Hz}$, while a $610 \mathrm{~Hz}$ susceptibility signal was obtained from a secondary coil wrapped directly around the semple. The pressure was generated in a $1: 1$ fluid mixture of n-pentare and isormyl alchol contained inside a 3.2 nrm diameter Teflon cup enclosed incide a berylium-copper cell, eliployiny the self-clamped technifue. The pressure was monitored by a lead manmeter with an accuracy of \pm 1 khar. A superconducting magnet capable of yenerating a magnetic field up to OT was employed for the magnet ic field studies.

The temperature dependerce of the ac maynetic susceptibility at various values of pressure is lisplaved in Fig. 5.[24] The increase of the susceptibility below "ugk at "ilitent pressure, in accordance with previous 
reports, $[10,11]$ indicates tile ons of terromagnetic correlabiuis. sumilar to other itinerant ferromagne-;, [16] $x$ decreases with increasing pressure. Also shown in the figure is the increase of the superconducting transition temperature with increasing pressure, consistent with the observation that magnetism, which depresses $T_{s}$, is suppressed by the applied pressure.

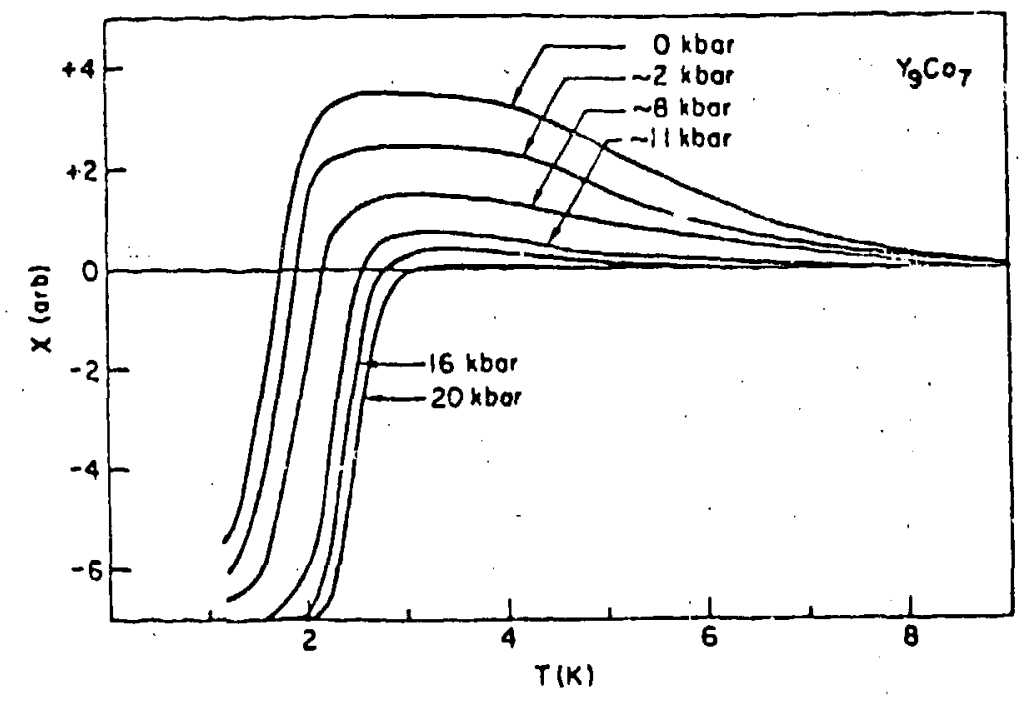

Fig. 5 Magnetic susceptibility, $x$, vs $T$ at various pressure values.

Figure 6 shows the temperature dependence of electrical resistance at various pressures. I : is particularly intriguing to observe that the resistance is independent of pressure up to $\sim 20 \mathrm{kbar}$ for $T>8 \mathrm{~K}$. This pressure independence seems to indicate that the magnetic correlations exist only for $T<8 K$. As can be seen, the higher the pressure, the sharper the transition, very similar to the nressure dependence of $x$ near the transition shown in Fig. 5. This interesting experimental result indicates that, the ferromagneitic correlations and superconducting fluctuations at ambient pressure, co-exist around $T$ but vary spatially (the "hybrid" state), in agreement with suggestions in Refs. 10 and 11. Furthermore at high pressure, the magnetism is suppressed and the sample becomes more "uniform" spatially, giving rise io a sharper transition.

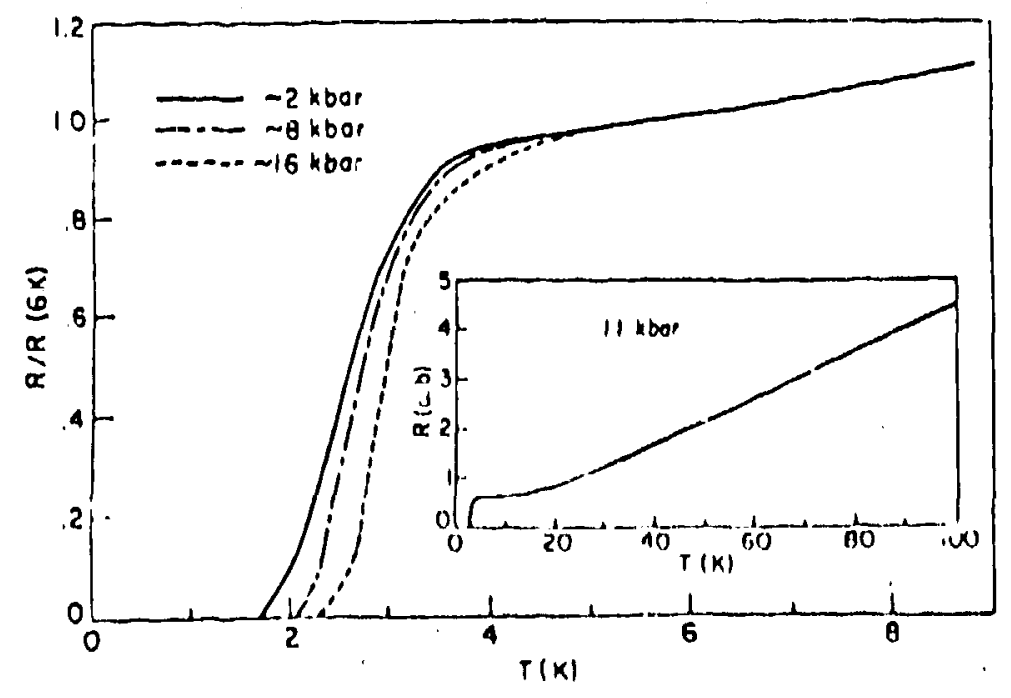

Fig. 6 Resistance, R, (with respect to $R$ ai $6 K$ ) vs $T$ at $\approx 3$ kbar, $\approx 8$ kbar, and $216 \mathrm{kbar}$. I is et shows $R$ vs $T$ at $\sim 11$ kbar.

W' have also medsured the resiitance as a function of an external maynet ic field up to $6 \mathrm{~T}$. Figure 7 exhibits some of our data at w $15 \mathrm{kbar}$ and Fia. \& demonstrates the field dependence of $R$ at $1.25 \mathrm{~K}$ and $2.5 \mathrm{~K}$ at various villues of prossure. It is striking to note that the maynetoresistance for messures $>6$ khat is alwas unsitive for $i<4.2 \mathrm{~K}$. in constrast to the ambient Hesside result that the mayneteresistance is neyative. [10] However, this field resendence is in accorilance with an earlier conclusion that pressure 
suppresses magnetism thus reducing the (negative) magnetoresistance. In want of an appropriate theory for the critical field of a magnetic superconductor, we define the midpoint of the transition as the upper critical field, $H_{\text {c }}$ (following Ref. 17). Figure 9 shows $H_{C}$ vs $T$ at various values of pressure. As expected, $\mathrm{H}_{\mathrm{c} 2}$ increases with increasing pressure.

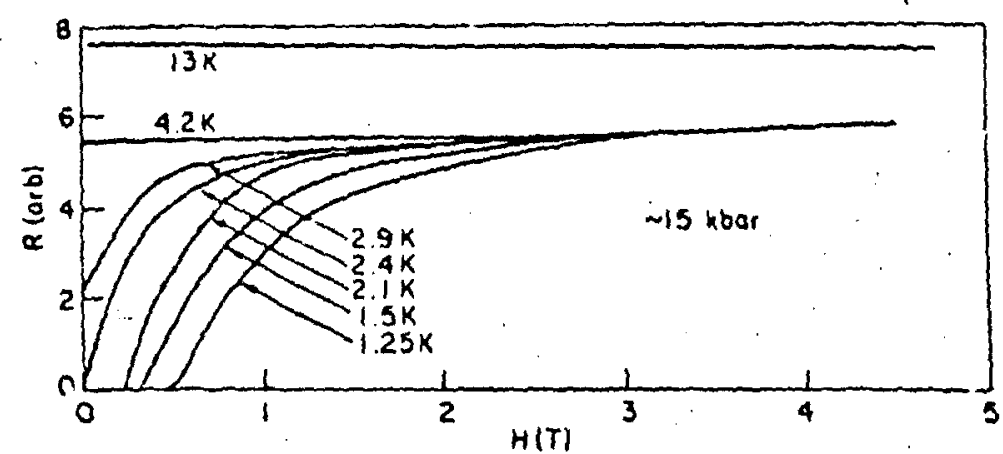

Fig. 7 Resistance, $R$, vs the magnetic field, $H$, at various temperatures at 15 kbar.

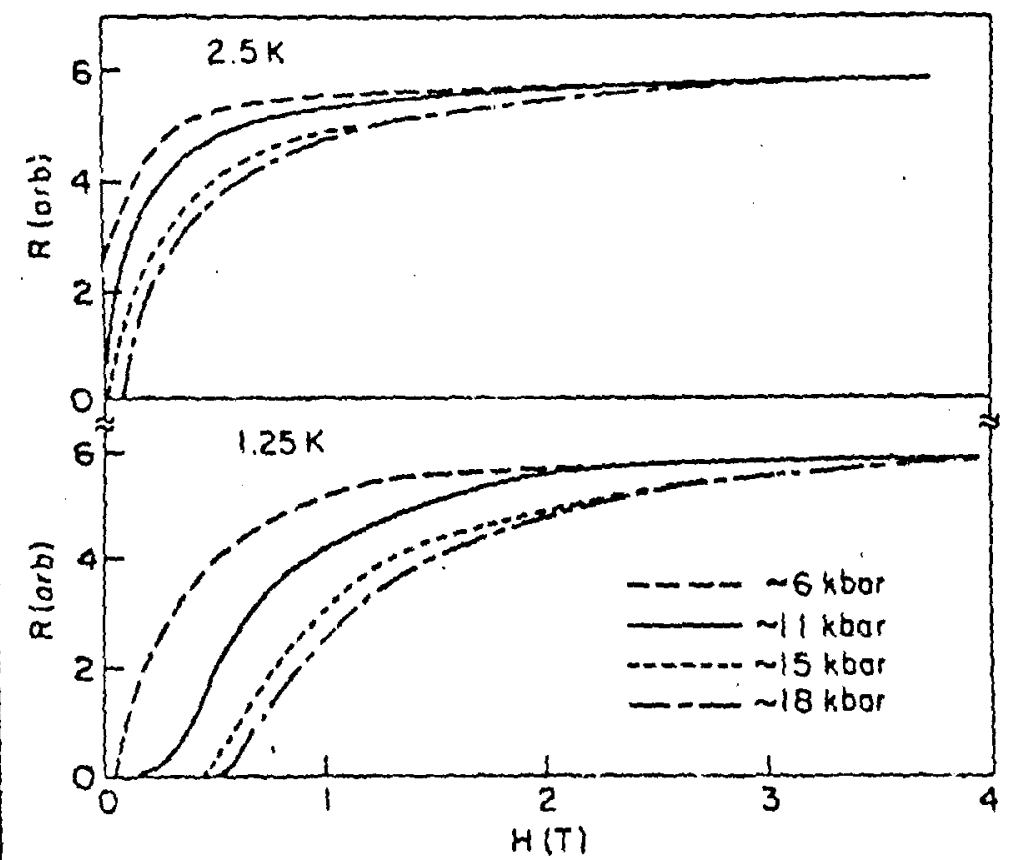

Fig. 8 Resistance, R, VS $H$ at various values of pressure and temperature.

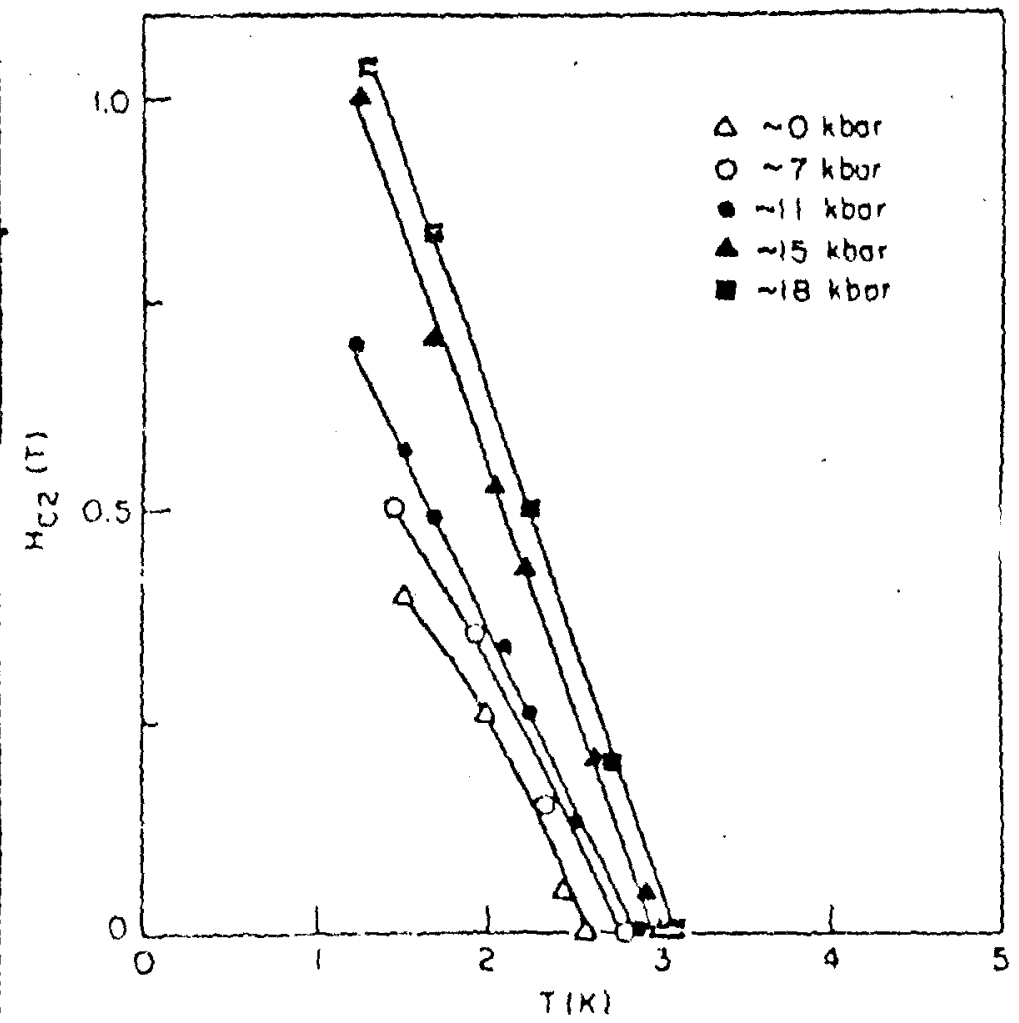

Fig. 9 Critical field, II vs $T$ at various vilues of pressure. 
Our high pressure experiment has yielded the following results: a) high pressure strongij suppresses magnetisil; b) the magnetism is itinerant; c) $T_{s}$ and $H_{1}$ increase with pressure; d) the superconducting transition is sharper at higher pressures; ej the magnetores istance for pressuie greater than 8 kbar and temperatures lower than $5 \mathrm{~K}$ is a! ways positive; and f) the ferromagnetic correlations and superconducting fluctuations co-exist around $T$ in the magnetic region and they vary in space. 'It is interesting to note that ume of these resul is can be interpreted in terms of Fig. I in which the superconducting transition, $T_{\text {s }}$, is depressed because of the presence of magnetism. Upon the application of high pressure, magnetic interactions are suppressed, thus raising $T_{s}$ toward $T_{50^{\circ}}$ This result siggrasts the need to repeat our experiments at much higher pressure.

The question of the possible co-existence of superconduciivity and maglietic correlations, as hinted by the neutron scattering results discussed above, has to be answered. A better reutron scattering experiment is obviously necessary; this requires the preparation of single crystal $\mathrm{Y}_{9} \mathrm{Co}_{7}$. Furthermore, microscopic studies such as NMR, muon spin resonance and relaxation, ultrasonic attenuation, tunneling, microwave impederice, and the MUssbauer effect are needed to understand the intriguing physical properties in $\mathrm{Y}_{9} \mathrm{CO}_{7}$.

Beyoild doubt, the band calculations are essential. In order to understand $\mathrm{Y}_{9} \mathrm{CO}_{7}$, a detailed theory taking into account the magnetic correlations of the $3 d-e l e c t r o n s$ of $C_{0}$ and the superconducting pairing in the $4 \mathrm{~d}$-electrons of $Y$ should be vital. It is also important for the theory to go beyond the mean field approximation to include superconducting fluctuations and elertromagnetic effects [3] and to calculate the physical quantities obtalnable from the experiments suggested above.

ACKNOWLEDGEMENTS - We would like to thank Drs. K. Machida and B.V.D. Sarkissian for many stimulating discussions. Two of us (CYH and CEO) are supported by the US Department of Energy and another (CST) is supported in part by the Office of Naval Research and the Energy Laboratory of the University of Houston.

\section{REFERENCES}

1. Ternary Superccnductors, G.K. Shenoy, B.D. Dunlop, and F.Y. Fradin, eds. (North, Holl and, 1980).

2. Superconductivity $i$, Ternary Compounds II, M.B. Maple and 0 . Fiscier, eds. (Springer Verlag, Berl in, Heidelberg, 1982).

3. H. Matsumoto and H. Umezawa, Cryogenics 23, 37 (i983).

4. A. Kolodziejczyk, B.V.D. Sarkissian, and B.R. Coles, J. Phys..F10, L333 (1980).

5. E. Gratz, H.R. rirchmayer, V. Sechovsky, and E.P. Hohlfarth, J. Mag. Mag. Mat. 21, 191 (1980).

6. J. Sebek, J. Stehnn, V. Sechovsky, and E. Gratz, Sol. State Commun.

7. $40,457(1981)$.

7. E. Gratz, J.O. Strom-01sen, and M.J. Zuckermann, Sol. State Commun. 40,833 (1981).

8. B.V.D. Sarkissian, A.K. Grover, and B.R. Coles, Phys ica $B+C \underline{109}$ and $\underline{110}, 2041$ (1982).

9. W. Cheng, G. Creuzet, P. Garoche, I.A. Campbell, and E. Gratz, J. Phys. F12, 475 (1982).

10. B.V.U. Sarkissian in Superconductivity in $d$ - and f-Band Metals, $W$. Buckel and $W$. Weber, eds. (Kernforschunyeszentrum Karlsruh.? 1982) 
Pp. 311; B.V.D. Sarkissian and A.K. Grover, J. Phys F1?, Li07 (198?).

12. A.K. Grover and B.V.D. Sarkissian, J. Mag. Mag. Mat. 31-34, 515 (1983).

13. A. van de Liet, P.H. Frings, A. Menovsky, J.J.M. Franse, J.A. Mydosh, and G.J. Nieuwenhuys, J. Phys. F12, L153 (1982).

14. C.Y. Huang, C.E. Olsen, W.W. Fülier, J.H. Huang, and S.A. Wolf, Sol. State Cormun. 45,795 (1983).

15. D.W. Harrison, K.C. Lim, J.D. Thompson, C.Y. Huang, P.D. Hambourger, and H.L. Luo, Phys. Rev. Lett. 46,280 (1981).

16. M.K. Wu, C.W. Chu, J.L. Smith, $\bar{A}$. L. Giorgi, C.Y. Huang, B.T. Matthias, and F.E. Wang, Sol. State Commun. 24, 507 (1980).

17. C.Y. Huang, D.W. Harrison, S.A. Wolf, W.W. Fuller, and H.L. Luo, Physica $B+C 109$ and 11C, 1649 (1982).

18. - H. Nakanishi, K. Machida, and T. Matsubara, Sol. State Commun. 43,899 (1982).

19. X.L Lef, C.S. Ting and J.L. Birman, preprint.

20. R. Lamaire, J. Schweizer, and J. Yakinthos, Acta-Cryst. B25, 710 (1969).

21. A.K. Grover, B.R. Coles, B.V.D. Sarkissian, and H.E.N. Stone, J LessCommon Met. 86, 29 (1982).

22. A.E Ray, A Review of the Binary Rare Earth-Cobalt Alloy Systems, COBALT; 1974 , No. 1; pp. 13.

23. Similar results have been obtained in Ref. 13.

24. In Ref. 13, $x$ was measured up to 4.7 kbar. 